Bundesgesundheitsbl - Gesundheitsforsch Gesundheitsschutz 2007 ·50:128-129 DOI 10.1007/s00103-007-0133-2 (c) Springer Medizin Verlag 2006

\title{
Mitteilung des Robert Koch- Instituts zur Aufnahme von Reinigungs- und Desinfektions- geräten in die Liste der geprüften und anerkannten Desinfektionsmittel und -verfahren gemäß § 18 IfSG
}

Die Prüfungen beinhalteten keinen quantifizierbaren Test der Reinigungsleistung und auch keine weiteren Kriterien zur Verfahrensführung, die über die o.a. hinausgehen. Aus der Eintragung kann auch keine generelle Eignung des Desinfektionsverfahrens für spezielle Medizinprodukte, die geeignete Adaptoren erfordern, wie z. B. Hand- und Winkelstücke, abgeleitet werden.

Im Juli dieses Jahres wurden die ersten Teile der Norm für Reinigungs- und Desinfektionsgeräte DIN EN ISO 15883 $[3,4]$ veröffentlicht. Diese von der EUKommission mandatierte Norm dient der Umsetzung des Medizinproduktegesetzes. Nach Inkrafttreten der Norm ist davon auszugehen, dass nur noch Geräte, die diese Norm erfüllen, ein CE-Zeichen als Voraussetzung der Verkehrsfähigkeit als Medizinprodukt erhalten.

Diese Norm beinhaltet überwiegend andere Prüfkriterien (z.B. auf die Reinigungsleistung ausgerichtete) als die für die Eintragung in die o. a. Liste erforderliche Prüfung. Zum Beispiel ist eine mikrobiologische Prüfung in der Norm nicht zwingend vorgesehen. In $\bullet$ Tabelle 1 sind die Anforderungen für die Eintragung in die RKI-Liste im Vergleich zu den entsprechenden Anforderungen der Norm dargestellt.

Aus dieser Gegenüberstellung geht hervor, dass die verschiedenen Ziele von
Norm und RKI-Liste unterschiedliche Anforderungen bedingen. Die Verfahren entsprechend der o. a. Liste sind für behördlich angeordnete Desinfektionsmaßnahmen vorgesehen. Sie beinhalten erhöhte Anforderungen hinsichtlich der Sicherheit vor dem Austritt von Infektionserregern aus den Geräten. Dies ist jedoch nur in besonderen Situationen erforderlich, z. B. wenn Krankheitserreger auftreten, die in Deutschland nicht bzw. nicht mehr vorkommen und schwerwiegende Erkrankungen (z.B. Erreger hämorrhagischer Fieber) verursachen. Der Einsatz entsprechend ausgestatteter RDG ist somit in Einrichtungen angezeigt, in denen bestimmungsgemäß Patienten mit hochgefährlichen, ggf. importierten Infektionen behandelt werden (sofern hier nicht andere Desinfektionsverfahren, z. B. Dampfdesinfektion, Einlegeverfahren, Verbrennen, vorgegeben sind). In Deutschland sind dies z. B. die dafür benannten Kompetenzzentren bzw. vergleichbare Isoliereinheiten.

Die Anforderungen der Norm sind auf die routinemäßige Aufbereitung von Medizinprodukten ausgerichtet. Hierbei ist es in der Regel nicht erforderlich, auf eine Vorspülung bzw. Reinigung mit jeweils nachfolgendem Flottenablass zu verzichten. In Teil 2 der Norm [4], der die Anforderungen an RDG zur thermischen Desinfektion von chirurgischen 
Tabelle 1

\section{Vergleich der Prüfkriterien der RKI-Prüfung mit entsprechenden}

Anforderungen der DIN EN ISO 15883

\begin{tabular}{|c|c|c|}
\hline & RKI-Prüfung & DIN EN ISO 15883-1, -2 \\
\hline Prozessführung & $\begin{array}{l}\text { Kein Ablauf der Flotte vor Ende } \\
\text { der Desinfektionsphase: } \\
\text { - Keine Vorspülung bzw. } \\
\text { Reinigung mit Flottenablass } \\
\text { - Flotte darf nicht aus der } \\
\text { Maschine austreten } \\
\text { (z.B. an den Türen) } \\
\text { - Bei Programmunterbrechung } \\
\text { darf die Flotte nicht } \\
\text { abgepumpt werden }\end{array}$ & $\begin{array}{l}\text { Vorspülen und Reinigung mit } \\
\text { Flottenablass vor der } \\
\text { Desinfektionsphase möglich } \\
\text { - Austritt von Flotte darf Prozess- } \\
\text { ablauf nicht beeinträchtigen } \\
\text { (5.4.1.2) } \\
\text { - Keine entsprechende } \\
\text { Festlegung }\end{array}$ \\
\hline Überlaufsicherung & $\begin{array}{l}2 \text { unabhängige Sicherungen für } \\
\text { den Wasserzulauf, die verhindern, } \\
\text { dass das Überlaufsystem aktiviert } \\
\text { wird }\end{array}$ & Keine entsprechende Regelung \\
\hline $\begin{array}{l}\text { Verhinderung von } \\
\text { Toträumen }\end{array}$ & $\begin{array}{l}\text { Das flotteführende System darf } \\
\text { keine Leitungsabschnitte enthalten, } \\
\text { die nicht den Desinfektions- } \\
\text { bedingungen (Temperatur, Zeit) } \\
\text { ausgesetzt sind }\end{array}$ & $\begin{array}{l}\text { Rekontamination des Behand- } \\
\text { lungsgutes muss so weit ver- } \\
\text { hindert werden, wie es der } \\
\text { nachfolgenden Verwendung } \\
\text { des Gutes entspricht (5.3.1.1) } \\
\text { - Totraumvolumen und Reini- } \\
\text { gungsmethode für die Zufuhr- } \\
\text { leitungen und Ventile sollen } \\
\text { angegeben werden (5.5.1.2) }\end{array}$ \\
\hline Ablauf & $\begin{array}{l}\text { Der Ablaufpumpe muss ein Ventil } \\
\text { vorgeschaltet sein }\end{array}$ & Keine entsprechende Festlegung \\
\hline Abluft & $\begin{array}{l}\text { Durch die Abluft darf es nicht zur } \\
\text { Rekontamination kommen }\end{array}$ & $\begin{array}{l}\text { Durch die Abluft darf es nicht zur } \\
\text { Rekontamination kommen (5.24.5) }\end{array}$ \\
\hline $\begin{array}{l}\text { Trocknungs- } \\
\text { einrichtung }\end{array}$ & $\begin{array}{l}\text { Durch die Trocknungseinrichtung } \\
\text { darf es nicht zur Rekontamination } \\
\text { kommen }\end{array}$ & $\begin{array}{l}\text { Rekontamination des Behand- } \\
\text { lungsgutes muss so weit ver- } \\
\text { hindert werden, wie es der } \\
\text { nachfolgenden Verwendung } \\
\text { des Gutes entspricht (5.3.1.1) } \\
\text { - Qualität der Trocknungsluft } \\
\text { darf weder Reinheit der } \\
\text { Beladung beeinträchtigen noch } \\
\text { sie mikrobiologisch kontami- } \\
\text { nieren (4.5.3) }\end{array}$ \\
\hline Temperaturmessung & Flotte & Beladung und Kammerwände \\
\hline $\begin{array}{l}\text { Prüfung der } \\
\text { Reinigung }\end{array}$ & Keine quantifizierbare Prüfung & $\begin{array}{l}\text { zwei Prüfungen: Prüfanschmut- } \\
\text { zung gemäß DIN EN ISO } 15883 \\
\text { Teil } 5 \text { und Gegenstände aus nor- } \\
\text { malem Gebrauch (6.10) }\end{array}$ \\
\hline $\begin{array}{l}\text { Mikrobiologische } \\
\text { Prüfung }\end{array}$ & esinfektion & $\begin{array}{l}\text { Wahlweise zur Prüfung der } \\
\text { Reinigung }\end{array}$ \\
\hline
\end{tabular}

Instrumenten, Anästhesiegeräten u. Ä. beinhaltet, wird darauf verwiesen, dass in diesen Geräten Verfahren mit einem $\mathrm{A}_{\mathrm{o}}$-Wert von 3000 möglich sein müssen. Das entspricht einem Verfahren bei einer Temperatur von $90^{\circ} \mathrm{C}$ mit einer Haltzeit von 5 Minuten. Auch bei Desinfektionsverfahren, die nicht auf behördliche Anordnung erfolgen, ist es sinnvoll, diese Bedingungen anzuwenden, insbesondere aus Gründen des Personalschutzes bzw. für Medizinprodukte, die nicht anschließend sterilisiert werden.

Anträge zur Aufnahme von RDG in die RKI-Liste ruhten bis zum Erscheinen der oben angegebenen Norm. Sofern RDG die Anforderungen der Norm erfüllen, ist es für den Routinebetrieb nicht erforderlich, sie zusätzlich nach den Kriterien der RKI-Liste zu prüfen.

\section{Literatur}

1. Richtlinie des Bundesgesundheitsamtes zur Prüfung von thermischen Desinfektionsverfahren in Reinigungsautomaten. Bundesgesundhbl 23 (1980): 364-367

2. Kommentar zur Richtlinie des Bundesgesundheitsamtes zur Prüfung von thermischen Desinfektionsverfahren in Reinigungsautomaten. Bundesgesundhbl 35 (1992): 536-537

3. DIN EN ISO 15883 Reinigungs- und Desinfektionsgeräte - Teil 1: Allgemeine Anforderungen, Begriffe und Prüfverfahren, Deutsche Fassung EN ISO 15883-1:2006 DIN Deutsches Institut für Normung e.V. Beuth Verlag GmbH, 10772 Berlin

4. DIN EN ISO 15883 Reinigungs- und Desinfektionsgeräte - Teil 2: Anforderungen und Prüfverfahren von Reinigungs- und Desinfektionsgeräten mit thermischer Desinfektion für chirurgische Instrumente, Anästhesiegeräte, Gefäße, Utensilien, Glasgeräte usw., Deutsche Fassung EN ISO 158832:2006 DIN Deutsches Institut für Normung e.V. Beuth Verlag GmbH, 10772 Berlin 